\title{
Multimedia as Mediator of Knowledge between Older Generations and Present-Day Students of Art and Design
}

\author{
Nuno Martins \\ Polytechnic Institute of Cavado and \\ Ave / ID+ / FBAUP \\ Inês Calado \\ Polytechnic Institute of Cavado and \\ Ave
}

\author{
Heitor Alvelos \\ ID+ / Universidade do Porto
}

\author{
Abhishek Chatterjee \\ ID+ / Universidade do Porto
}

\begin{abstract}
This paper presents two studies constituted within two funded design research projects that are intended to develop tools which can support the transfer of specialized knowledge between an older generation of art, craft, and design practitioners and present-day students of art and design. The first study concerns a digital archiving and education platform designed to promote both research and practice in the area of traditional Portuguese manufacturing. It aims to aid in the conservation and continuity of local cultural and industrial heritage with a vision to ensure an effective access to the associated skills, techniques, and empirical wisdom for present and future patrons. The second study is about an interactive infographic that aims to synthesize and unravel complex systems of information relative to the creative and academic legacies of retired art and design teachers. The work is based on the documentation and analysis of testimonies from 30 key individuals, wherein, relevant information has been extrapolated and structured within the framework of an educational resource aimed for use in design research and pedagogy. Both studies seek to build knowledge-based connections between distinct generational and disciplinary segments. The respective interfaces are also meant to showcase the criticality of digital media in the rescue and re-inscription of artistic and creative legacies, and for fostering greater possibilities.
\end{abstract}

\section{CCS CONCEPTS}

- Applied computing; • Education; • E-learning;

\section{KEYWORDS}

Multimedia, E-learning, Digital Design, Infographics, Communication Design, Education

\section{ACM Reference Format:}

Nuno Martins, Heitor Alvelos, Abhishek Chatterjee, Inês Calado, and Mariana Quintela. 2020. Multimedia as Mediator of Knowledge between Older Generations and Present-Day Students of Art and Design. In 2020 The 4th International Conference on Education and Multimedia Technology (ICEMT 2020), July 19-22, 2020, Kyoto, Japan. ACM, New York, NY, USA, 6 pages. https://doi.org/10.1145/3416797.3416827

\section{(c) (†)}

This work is licensed under a Creative Commons Attribution International 4.0 License. ICEMT 2020, Fuly 19-22, 2020, Kyoto, fapan

(C) 2020 Copyright held by the owner/author(s).

ACM ISBN 978-1-4503-8837-5/20/07.

https://doi.org/10.1145/3416797.3416827

\author{
Mariana Quintela \\ Polytechnic Institute of Cavado and \\ Ave
}

\section{INTRODUCTION}

This article aims to showcase digital media's potential towards making meaningful and sustainable knowledge-based connections between an older generation of creatives and their younger counterparts in today's artistic field. In this regard, two design research studies which aim to adopt digital media as a strategic means for the reinscription of creative legacies, become the starting locus. The studies are being conducted under funded design research projects, namely Anti-Amnesia (AA) and Wisdom Transfer (WT), and involve researchers from The Research Institute for Design, Media and Culture [ID+] in Portugal.

This article begins by contextualizing the AA and WT projects, explaining their objectives alongside the corresponding conjectures on which the frameworks of the studies are based. In the case of AA, an analysis of the current state of the Portuguese handicraft industry is presented, leading to inferences on the relevance of digital media as a means of revitalizing the associated industrial and cultural heritage. In the case of the WT, the article argues the importance of infographics as an effective means of translating complex historical, cultural, and artistic information derived from oral histories into educational resources. The article concludes by describing the rationale and processes behind the development of an e-learning platform and a digital interactive infographics tool that serve as potential solutions.

\section{PROJECTS ANTI-AMNESIA AND WISDOM TRANSFER}

The design research and mediation project Anti-Amnesia (AA) aims to investigate the agency of design in the regeneration and reinvention of material, social, and cultural narratives surrounding disappearing traditional Portuguese craft and small-scale industries. Under the ambit of this project, a study, as an experimental digital design component, looks to explore the feasibility of a platform in web format, that brings together archiving, interdisciplinary collaboration, and e-learning, as a potential solution to address the decline of traditional making [1, 2].

Named 'Craft Academy', the study aims to:

- develop methodologies, through digital means and tools, that can contribute to the preservation of the industrial and cultural heritage of craft industries;

- contribute to the sustainability of the crafts through ensuring a continuous inter-generational and interdisciplinary transmission of skills, techniques and craft knowledge; and 
promote further research in the area of Portuguese manufacturing;

- make related knowledge and information more accessible;

- promote craft learning in order to encourage younger generations to recover and keep alive age-old practices as a way of valuing tradition.

Regarding Wisdom Transfer (WT), a parallelly running design research project at $\mathrm{ID}+$, the main goal is to register and potentiate the contributions of retired art and design professors towards their respective fields and creative pedagogy. The project results from the finding that in Portugal there is an insufficient usage of the wealth of knowledge and experience embedded in the oral histories of the retired teachers. It subsequently raises the hypothesis that the legitimization of such practice-based empirical wisdom in academic contexts can greatly extend the mastery and depth of the related disciplines.

Correspondingly, in a similar vein to AA, WT too has incorporated a digital design study component which focuses on building a digital solution that can improve the comprehensibility and access to WT's generated database of heterogenous anecdotal evidence. The study consists of a meticulous analysis of the collected information, followed by synthesis and presentation in an interactive infographic format. The infographics will subsequently be available on the WT project website for public dissemination.

\section{ANTI-AMNESIA AND THE CHALLENGES TO PORTUGUESE HANDICRAFT INDUSTRY}

The Industrial Revolution in the 18th century marked a period of profound change, with the transition from artisanal to industrial production methods. These changes brought about major economic and social changes throughout Europe. Craft production in the preceding eras was, essential to sustain artisanal communities. However, the transformations that took place, concurring with the massification of forms of marketing and distribution, led to the breakdown of the processes of craft production [3].

Craft industries in Portugal are diverse and closely linked to popular culture, particularly in rural areas, however, many techniques and practices are in rapid decline due to the growing migratory exodus of rural folk to national and international urban centers.

During the 20th century, the strong growth of industrialized production, entailing lower costs and higher profitability in comparison to manual crafts, led to the rise of consumer demand for machine made goods and signaled the gradual decline of craft-based businesses. The circumstances also resulted in a decreasing of interest in younger generations to consider careers in craft-based professional activities, which became a major factor in the disappearance of several traditional techniques and skills [3].

However, craft activity has survived in some contexts, especially in niche markets where craft communities have been able to maintain their commercial relevance. Additionally, due to, the late onset of the industrial revolution in Portugal, various craft industries had an extended run and became essential constituents of Portuguese lifestyle and culture. Since the 1980s, European programmes to support and encourage the development of local economies have contributed to the enhancement of the artisanal heritage and the strengthening of local and regional identity of peoples, while promoting the study and recognition of traditional craft activities and products understood as repositories representative of the collective identity of communities [3].

In Portugal the turning point for the recognition of these industries, as a sector of economic activity with important and relevant cultural value, happened during this time period along three critical milestones: the legal and political acknowledgement by the government; recognition by the civil society through the creation of support structures; and the emergence of associations and cooperatives.

Major impetus was also drawn from the creation of PPART - Programme for the Promotion of Crafts and Small Craft Enterprises which focused on the identification of craft activities and practitioners, with the goal of valuing traditional making. The programme made a decisive contribution to the sustenance of typical crafts by defining incentive policies for the sector, and by recognizing arts and crafts as a means of preserving important cultural values.

Handicraft is thus an enduring means for boosting the economy, tourism, and local employment, and retains potential towards increasing levels of territorial competitiveness of an economic, social, cultural and environmental nature [3]. The cultural heritage embodied in traditional crafts is an integral part of any nation and reflects the regional culture and tradition, and is explained by UNESCO as "our legacy from the past, what we live today and what will be passed on to future generations" [4].

In the present day, there is a wide range of traditional Portuguese industries and crafts, especially in the northern and central regions of the country, who face fundamental challenges to their recognition and longevity, largely due to new production models, rapid changes in consumption behaviors, and a public absence of the associated narratives.

Digital media can provide key resolutions in this regard by implementing technological measures that can preserve and promote craft traditions and techniques, and in consequence stimulate new activities and employment opportunities, especially in local and rural communities, thereby aiding in their sustainable development [5-7].

The shift to a digital future has not been a linear process for the craft industries, as its transition compromises factors such as the authenticity of the practice; the expertise built from generation to generation on an individual and community basis; the local or regional cultural identities, customs and practices; and, critically, the means of livelihood in the absence of conditions to carry out the practice.

AA, in this regard, engages with four traditional industrial practices in Portugal, in order to understand the factors behind their ongoing decline, and to determine the scope of digital technology towards making restorative impacts. The case studies include Almalaguês hand weaving, Azulejos tilemaking; small-scale footwear manufacturing; and letterpress printing.

\subsection{The 'Craft Academy' Online Platform}

The technological solution 'Craft Academy' that is being developed within this project aims to create a knowledge network concerning 


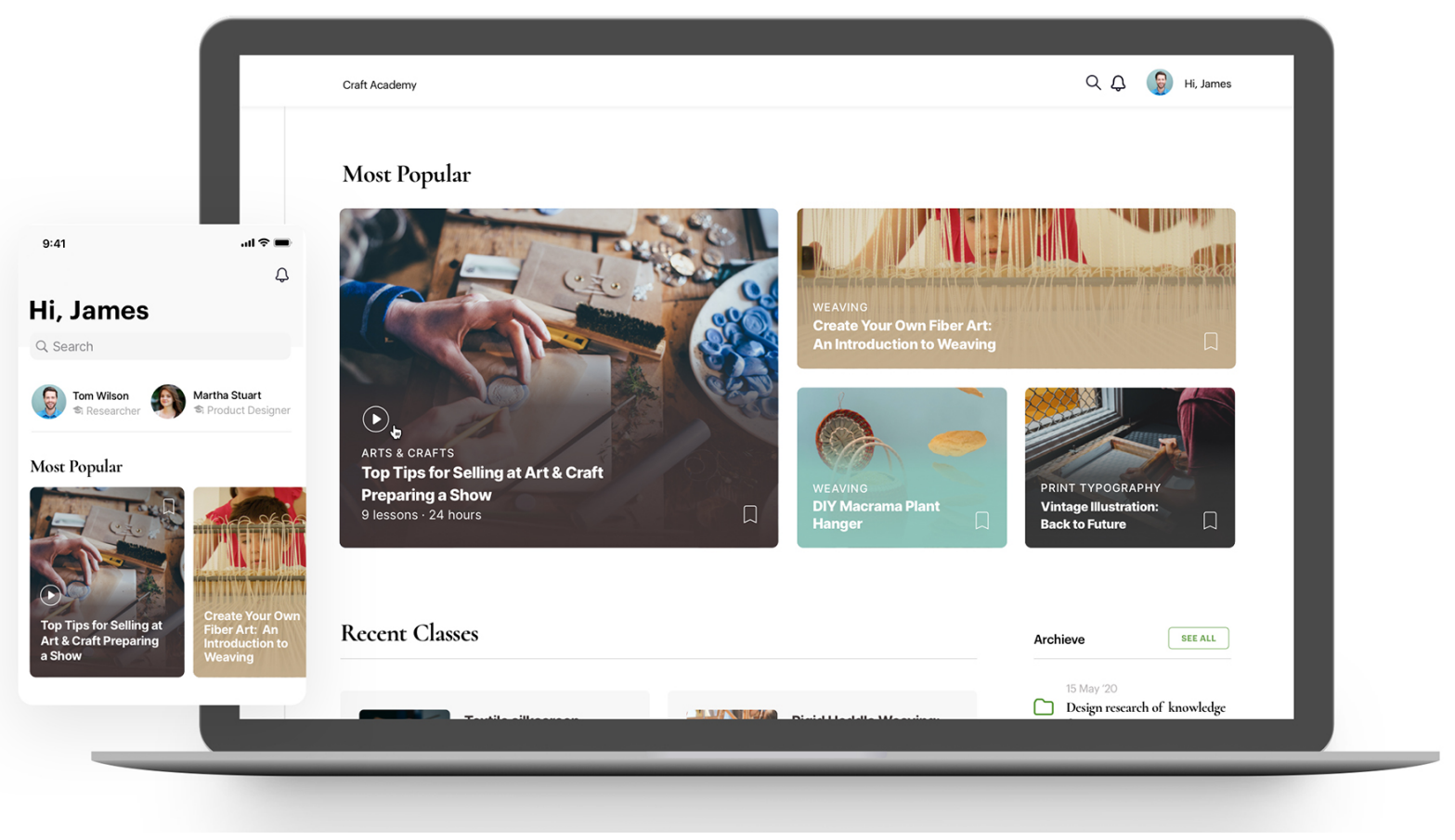

Figure 1: Design of the 'Craft Academy' online platform

crafts that fosters an exchange of ideas, possibilities of collaborations and partnerships, and a co-exploration of new creative models, product segments, and market opportunities between craft and design.

The online platform is especially directed towards a younger generation of active practitioners, students, researchers, designers, and other entities interested in the field, and intends to function as an e-learning and cooperative support infrastructure towards creating and disseminating digital content pertaining to traditional crafts. UNESCO [4] acknowledges that ensuring public accessibility to cultural heritage, including antiquated craft and other industrial practices, is a highly relevant global issue, which will allow the cultural traditions of a country or a region to not fall into disuse. Increasingly thus, individuals, organizations, and communities are using digital technologies to document and express what they create and value [4].

Digitization and online accessibility of cultural material, in addition to its conservational nature, enables a wider access to the material in the public domain for its usage in commercial and noncommercial use-cases.

A public domain status means that the material has no copyright protection and can be used without restriction because the copyright protection may have expired, or because the works have not yet been covered under copyright regulations. This is an additional issue which can be addressed by the online platform.

The method thus offers a multitude of educational opportunities essential for the development of craft industries in Portugal, seeking to augment cultural and creative capacities, towards consolidating crafts' relevance to contemporary creative economies and praxis.
The conclusion of this project is expected in the next months and will entail the development of a high-fidelity prototype according to the principles of UX Design. In figure 1 shows a preview of the online platform's design [8].

\section{WISDOM TRANSFER: AN INFOGRAPHIC TRANSLATION}

The second study, Wisdom Transfer (WT) project, is about an interactive infographic that aims to synthesize and unravel complex systems and dynamics of information pertaining to the creative and academic legacies of retired art and design teachers.

Infographics represent the human urge to understand and interpret the nature surrounding them. Old inscriptions, cave paintings, early maps, and scientific illustrations, all bear testimony to this instinct over the ages, and provide evidence of linkages between language and graphic content. It is also a reason why infographics are often used for educational purposes.

The goal is to assure that the legacy of wisdom and knowledge of the older generation can and will be passed on to the younger generations, with the aid of Multimedia, particularly through interactive infographics.

\subsection{Infographics as an Education Aid}

Infographic translation enables efficient and effective access to knowledge. Therefore, the concept of visual learning is applied to digital platforms largely because most audiences are "visual learners", as noted by Bradford [1], who estimates that up to $65 \%$ of the population falls into this category. 
Bradford also emphasizes the value of infographics as a pedagogical tool in making the correlation between design research and education.

Individual learning styles are still highly debatable, since it's been suggested that many variables have to be considered when it comes to the presentation of content, the quality of the teacher, and the field of study [9]. Even though it's important for learning experiences to factor in all variables, in the digital environment, however, visual engagement is highly dependent of the visual content, according to recent studies in digital marketing [10].

\subsection{Methodology}

In assembling a sound infographic system, the study has subscribed to a number of key graphic principles towards constructing the structure and defining the graphic content to be displayed in the platform. The principles that shape the infographic are therefore:

4.2.1 Not assuming that an infographic is always intuitive or selfexplanatory. Infographics typically rely heavily on the ability of their readers, and thus, when it comes to their construction, there's always a concern that the target audience might feel frustrated while engaging with the content due to an overbearing complexity of presented issues or a lack of contextual support. Sometimes an infographic can display a system that only people with a greater degree of knowledge of the subject may understand. To reduce this impact, it's important to explain how to read the infographic correctly by using a supplemental graphic or text [11].

4.2.2 Clarifying, and not just simplifying. In building infographics for complex subjects, graphic simplification might seem as an appealing strategy, however, it can compromise the reading experience. Because of the subtraction of content, the reader may not understand the full picture. It is thus pertinent to use maximum context support, and a further number of elements if necessary [11].

4.2.3 Recognizing the target and adapting the strategy. To start developing an infographic, it is advisable to recognize and gather information about the target audience. This allows the design team to create/adapt an infographic product in full knowledge of their specific needs and aspirations [12].

The infographics are being developed as a pedagogic tool catering to young students of art and design, who per usual exhibit a greater interest in independent arts and are more invested in education and technology overall.

4.2.4 Understanding which type of Infographics to use. According to Berinato [13], there are 4 types of information graphics: declarative, conceptual, exploratory, and data driven. Each type represents a distinct set of characteristics that dictate the best graphic elements to use.

The project accordingly has considered advancing under the criterion of declarative and data-driven infographics, modelling its approach based on the graphic exploration of the data collected, in order to determine the concept.

4.2.5 Understanding which type of Infographics to use. The study puts special emphasis on the use of space in infographics since it allows the target audience to comprehend the hierarchy of elements. Conventionally, elements that occupy more space in the visual layout, and have more contrast, display the most important information to retain. Likewise, there are a number of other aspects of the Gestalt Theory [14] that are being explored as a baseline for the structure and information architecture in the platform [12, 15].

The WT study is also exploring the use of blank space, mainly because of its relative value to alternative aesthetic elements, and the organization of the content set. The incorporated text thus has a bigger leading, permitting a more relaxed reading experience.

In the WT infographic, the information related to the retired art and design professors and researchers regarding their experience as students is hence placed in the same shell. This implies that the timeline pertaining to their student experience and the respective fields of education are within the same framework (figure 2).

Chapman [16] also advises that when organizing elements through the use of color, the elements of the same nature must use the same color to be correctly identified. Accordingly, in the WT project when using filters, the information concerning this change is presented in the previously determined color for the users to understand the filters implication. In the panel detailing the artists' experience as students, the students' field - identified in a label below - is displayed with the same color behavior for the readers to immediately comprehend the subjects' respective fields of study.

The project conceives that the use of images facilitates establishing a personal connection with the readers. In order to ensure and leverage such connection, Nielsen [17] suggests the use of profile pictures instead of stock photos, since it has proven to create more empathy with the audience, and an amplifying credibility. Also, when in contact with these simple photos, readers typically spend up to $10 \%$ more time looking at them than reading the descriptions.

The WT project hence uses profile images from each subject in order to derive greater subject affinity from readers.

4.2.6 Considering applicable UI \& UX Design elements. In digital environments the UI and UX fundamentals help users navigate through the interaction process. Visual elements should thus be carefully thought out to gently guide users and make them enjoy the interaction with the platform [18].

In this regard the WT study makes it a point to be consistent with its design choices. Buttons are kept at a medium size, appealing to younger target audiences, and yet remaining readable for older viewers. Also, they are placed in designated areas for easy localization. When deactivated, their color becomes more discreet, however, when reactivated, they present the originally assigned color. When it comes to filtering information in the platform, it's important to have a navigation set that helps readers go back to the main page. This allows them an understanding of the provided navigational paths.

Consequently, a key visual clue for viewers that enter the filter mode in the WT platform is a "back to overview" button that assures that they can go back to the infographic's initial state at any stage. When entering a different panel, like a summary of a particular artist or sample images of the associated works, the option to close the panel is made clearly visible. If the reader has further interest in following the artist's work or viewing a more complete version 


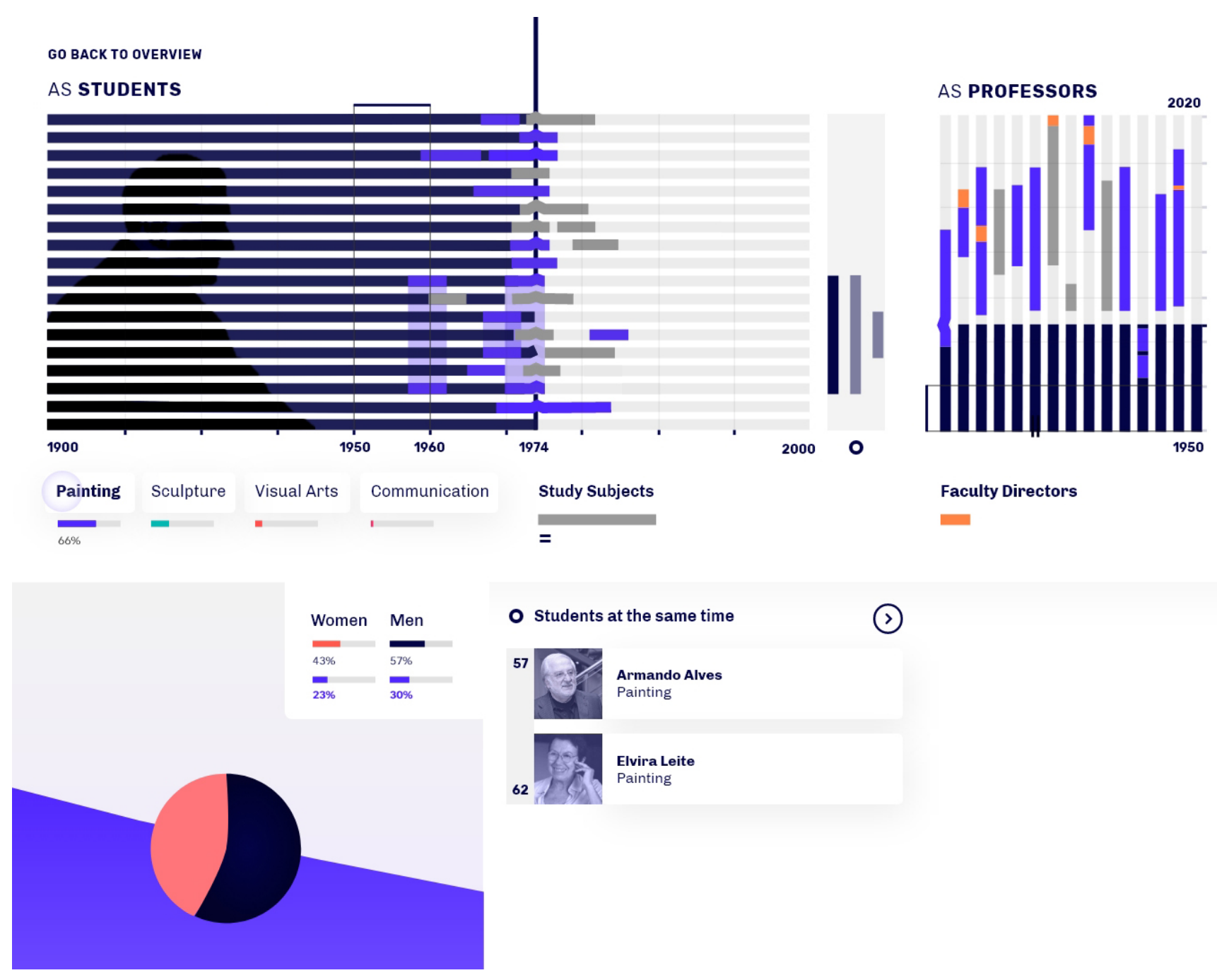

Figure 2: Wisdom Transfer infographic

of the related biography, there are buttons at hand that open up links to their full biography or website, in order to complement the webpage.

\subsubsection{Introducing visual Storytelling to engage with the audience.} To communicate information with greater efficiency, the project designers have used visual storytelling as a strategy to enhance the readers' emotional connection and response [19].

The technique generally entails: the use of visual content like illustrations or animations to conduct a story or perceive data; the use of an emotionally appealing storyline; and an equally emotive color scheme [12]. The WT infographic platform, accordingly, is in the process of incorporating visually stimulating graphics and illustrations as a way to draw attention to the sequential timeline detailing the life stories of the researched artists. The objective, similar to the inclusion of the profile picture, is to draw greater affinity and interest from the readers regarding the presented personalities [20].

\section{CONCLUSIONS}

The article intends to demonstrate that the older generations of creative practitioners embody knowledge and experience of great contemporary relevance, which is worthy of further inscription, and should be seen as intellectual resources for passing on to future generations. This knowledge-based heritage is currently at a risk of permanent loss if more conservational and restorative initiatives are not developed.

In this process of knowledge transfer, digital media can play an important role, especially because newer generations are more used to technological tools and digital solutions.

The two projects presented in this article are still under development and are expected to be concluded by the end of September 2020. The next and final phase of the study will be dedicated to usability testing. Depending on the results obtained, the necessary improvements will be made in order to ensure a high level of usability of online platforms. 
In conclusion, it should be emphasized that although the studies are focused towards the creative fields, the assayed problems and solutions can be applied to and explored in different fields of knowledge.

\section{ACKNOWLEDGMENTS}

The authors wish to thank the Foundation for Science and Technology, Portugal, for financing the Wisdom Transfer project (ID+/Unexpected Media Lab: POCI-01-0145-FEDER-029038; 20182020) and Anti-Amnesia project (ID+/Unexpected Media Lab: POCI01-0145-FEDER-029022; 2018-2020).

\section{REFERENCES}

[1] Bradford, W. C. 2011. Reaching the Visual Learner: Teaching Property Through Art. (September 1, 2011). The Law Teacher Vol. 11, 2004. Available at SSRN: https://ssrn.com/abstract $=587201$

[2] Poce A., Agrusti F., Re M.R. 2018. Heritage Education and Initial Teacher Training: an International Experience. Journal of E-Learning and Knowledge Society. Vol. 14, No. 2, DOI: https://doi.org/10.20368/1971-8829/1488

[3] Regio Crafts. 2014. Crafts Innovation: Caracterização do Setor do Artesanato em Portugal. SUSDesign, Portugal.

[4] UNESCO. n.d. Concept of digital Heritage. Available at https://en.unesco.org/ themes/information-preservation/digital-heritage/concept-digital-heritage.

[5] Ranieri M., Bruni I. 2018. Promoting Digital and Media Competences of pre- and in-Service Teachers. Research Findings of a Project from six European Countries. Journal of E-Learning and Knowledge Society. Vol. 14, No. 2, pp 111-125. DOI: https://doi.org/10.20368/1971-8829/1497

[6] Cheah, I., Phau, I. and Liang, J. 2015. Factors influencing consumers' attitudes and purchase intentions of e-deals. Marketing Intelligence \& Planning, Vol. 33 No. 5, pp. 763-783. DOI: https://doi.org/10.1108/MIP-05-2014-008

[7] Portugal Global. 2019. Artesanato Português - Tradição e Design na promoção do património, da cultura e da identidade de um povo. Portugal Global, Vol. 111, pp. 6-27.

[8] Martins, N., Alvelos, H., Barreto, S., Chatterjee, A., Penedos-Santiago, E., Lima, C \& Quintela, M. (2020) E-learning as a strategic solution for the preservation and revitalization of disappearing industrial cultures in Portugal. Advances in Human Factors in Training, Education, and Learning Sciences. AHFE 2020. Advances in Intelligent Systems and Computing, vol 1211. Springer, Cham. DOI: https: //doi.org/10.1007/978-3-030-50896-8 47

[9] Riener, C. \& Willingham, D. 2010. The Myth of Learning Styles. Change: The Magazine of Higher Learning, Vol. 42, No. 5, pp. 32-35, DOI: 10.1080/00091383.2010.503139

[10] Kienapple, B. 2019. Must-Know Visual Content Marketing Statistics For 2019 [Infographic]. Business 2 Community. Available at: https://www. business2community.com

[11] Cairo, A. 2019. How Charts Lie: Getting Smarter about Visual Information. W. W. Norton \& Company: Independent Publishers.

[12] Knaflic, C. N. 2015. Storytelling with Data: A Data Visualization Guide for Business Professionals. Hoboken, New Jersey: Jonh Wiley \& Sons Inc.

[13] Berinato, S. 2016. Good Charts: The HBR Guide to Making Smarter, More Persuasive Data Visualizations. Boston, Massachusetts: Harvard Business Review Press.

[14] Wertheimer, M. 1923. Laws of organization in perceptual forms. First published as Untersuchungen zur Lehre von der Gestalt II, in Psycologische Forschung, 4, 301-350. Translation published in Ellis, W. (1938). A source book of Gestalt.

[15] Chaparro, B. et al. 2004. Reading Online Text: A Comparison of Four White Space Layouts. Usability News. Vol. 6 No. 2.

[16] Chapman, C. 2020. Exploring The Gestalt Principles Of Design. Toptal.

[17] Nielsen, J. 2010. Photos as Web Content. NN Group. Available at: https://nngroup. com/articles/photos-as-web-content

[18] Martins, N., Campos, J., Simoes, R. 2020. Activerest: Design of A Graphical Interface for the Remote use of Continuous and Holistic Care Providers. Advances in Science, Technology and Engineering Systems Journal, vol. 5, no. 2, pp. 635-645. DOI: http://dx.doi.org/10.25046/aj050279

[19] Martins, N., Enes, J. 2020. Spot Toyota: Design and Development of a Mobile Application for Toyota's Promotion Actions to the Young Audience. Advances in Science, Technology and Engineering Systems Journal, vol. 5, no. 3, pp. 469-477. DOI: http://dx.doi.org/ 10.25046/aj050358

[20] Martins, N., Barreto, S., Penedos-Santiago, E., Lima, C. \& Calado, I. 2020. The infographic process of synthesizing complex information about the individual legacies of retired teachers and researchers in art and design. Advances in Human Factors in Training, Education, and Learning Sciences. AHFE 2020. Advances in Intelligent Systems and Computing, vol 1211. Springer, Cham. DOI: https: //doi.org/10.1007/978-3-030-50896-8_6 